\title{
CAMBIOS FISICOQUÍMICOS Y SENSORIALES LIMITANTES DE LA VIDA DE ANAQUEL DE MANGO FRESCO CORTADO
}

\author{
PHYSICOCHEMICAL AND SENSORY CHANGES THAT LIMIT SHELF-LIFE \\ OF FRESH-CUT MANGOES
}

\author{
Rosa Ma. Salinas-Hernández ${ }^{1,3}$, María Élida Pirovani ${ }^{2}$, Alfonso A. Gardea-Béjar ${ }^{1}$ y \\ Gustavo A. González-Aguilar ${ }^{1 *}$
}

\begin{abstract}
${ }^{1}$ Centro de Investigación en Alimentación y Desarrollo, A.C. Km 0.6 Carretera a La Victoria, Hermosillo, Sonora (83000), México. ${ }^{2}$ Instituto de Tecnología de Alimentos, Universidad Nacional del Litoral. Paraje El Pozo, Santa Fe, Argentina. ${ }^{3}$ Universidad Juárez Autónoma de Tabasco, División Académica de Ciencias Agropecuarias. Km 25 Carretera Villahermosa-Teapa. 86000 Centro. Tabasco, México.
\end{abstract}

* Autor para correspondencia (gustavo@ciad.mx)

\section{RESUMEN}

Frutos de mango (Mangifera indica L.) cv. 'Haden' fueron procesados mínimamente y almacenados a $5{ }^{\circ} \mathrm{C}$ durante $14 \mathrm{~d}$. A intervalos de 2 d se determinaron las variables fisicoquímicas de color $\left[L^{*}, a^{*}\right.$ y $b^{*}, y$ los valores derivados: croma $\left(C^{*}\right)$, ángulo de matiz $\left(h^{\circ}\right)$ y diferencia total de color $\left.\left(\Delta \mathrm{E}^{*}\right)\right]$, así como sólidos solubles totales (SST), firmeza y pH. También se evaluaron los atributos sensoriales de olor característico, apariencia, brillo, color, sabor, textura, acidez y dulzor, mediante un panel entrenado. Se llevó a cabo un análisis de componentes principales (ACP) y análisis de regresión, para identificar las variables con mayor variabilidad durante el almacenamiento y estimar la tasa de cambio tanto de diferentes atributos sensoriales como de variables fisicoquímicas. El ACP indicó que la menor variabilidad del mango fresco cortado fue con respecto a $h^{\circ}$ y SST. Los atributos sensoriales con menor cambio fueron acidez, textura, dulzor y olor. El análisis de regresión indicó que el brillo presentó la mayor tasa de cambio, mientras que el olor característico tuvo la mayor duración en el producto durante el almacenamiento. Entre las variables fisicoquímicas, las de mayor y menor tasa de cambio fueron croma $\left(C^{*}\right)$ y firmeza, respectivamente. Los cambios fisicoquímicos y atributos sensoriales con mayor variabilidad y mayor tasa de deterioro, cambios críticos durante el almacenamiento, pueden usarse como indicadores del deterioro en estudios de vida de anaquel del mango fresco cortado.

Palabras clave: Mangifera indica, análisis multivariado, calidad sensorial, fruto fresco cortado.

\section{SUMMARY}

'Haden' mango (Mangifera indica L.) fruits were minimally processed and stored at $5{ }^{\circ} \mathrm{C}$ during $14 \mathrm{~d}$. At $2 \mathrm{~d}$ intervals, processed fruits were sampled to evaluate physicochemical variables of color $\left[L^{*}, a^{*}, b^{*}\right.$, and derived values: chroma $\left(C^{*}\right)$, hue angle $\left(h^{\circ}\right)$ and total color difference $\left.\left(\Delta \mathrm{E}^{*}\right)\right]$, as well as sensory attributes: sweetness, texture, brightness, color, flavor, odor and appearance, as perceived by a trained panel. Data were analyzed using principal components analysis (PCA) and regression to assess changes physicochemical variables and sensory attributes during storage.
Principal components analysis indicated that hue angle $\left(\mathrm{h}^{\circ}\right)$ and total soluble solids (TSS), and the sensory attributes of acidity, texture, sweetness and odor, showed a slight relevance on the variability observed on the sliced mango units during storage. Regression analysis indicated that brightness had the highest deterioration rate, whereas odor was the attribute with less change during storage. In the same way, $C^{*}$ was the physicochemical variable with the highest deterioration rate while the lowest deterioration was for firmness. Physicochemical and sensory changes with the largest variability and with the highest deterioration rates, are critical changes that can be used as spoilage indicators in shelf-life studies of fresh-cut mangoes.

Index words: Mangifera indica, multivariate analysis, sensory quality, fresh-cut fruits.

\section{INTRODUCCIÓN}

En los últimos años el segmento de mayor crecimiento dentro de la industria alimentaria es el de frutos y vegetales frescos cortados (FVFC), por lo que la investigación en este rubro se ha incrementado sustancialmente. Entre los frutos tropicales con mayor potencial en el mercado de FVFC se encuentra el mango (Mangifera indica L.), debido a su alta producción mundial, preferencia por el consumidor y alto valor nutricional (González-Aguilar et al., 2008). Los FVFC poseen las cualidades de los productos frescos recién cosechados (Alzamora et al., 2000), pero el pelado y cortado del fruto induce estrés en el tejido vegetal, lo que afecta los atributos sensoriales aún antes de que ocurran cambios fisicoquímicos o microbiológicos limitantes de la vida de anaquel (Gil et al., 2006).

Para evaluar la vida útil a partir de cambios sensoriales, es necesario determinar aquéllos que son críticos. En este sentido, el análisis sensorial descriptivo cuantitativo 
(ADC) es uno de los métodos más útiles, pues permite determinar la magnitud y el sentido de los cambios sensoriales en función del tiempo de almacenamiento (Sancho et al., 2002). Sin embargo, el ADC es complejo porque implica la formación de un panel capaz de identificar correctamente cada binomio descriptorsensación y cuantificar la magnitud del cambio en los distintos atributos (Sancho et al., 2002), por lo que a pesar de su efectividad como prueba sensorial, el ADC ha sido poco utilizado en la evaluación de FVFC (Qüesta et al., 2007).

Rivera-López et al. (2005) indican que los cambios fisicoquímicos y sensoriales en los FVFC difieren en función del tipo de producto, tratamiento, tipo de corte y temperatura de almacenamiento, por lo que es necesaria su evaluación en el producto de interés. En este sentido, el uso adecuado de métodos estadísticos y de análisis de datos puede ser útil para identificar los principales cambios en calidad. Entre las variables de respuesta consideradas en un experimento, algunas pueden variar poco o resultar de combinaciones lineales de otras variables, lo que aumenta el trabajo de discriminación pero sin incrementar la precisión en la interpretación de resultados (Barbosa et al., 2006). Por ello es necesario considerar la relevancia de las variables de respuesta en función de su variación, y descartar las de poca importancia para reducir el tiempo y recursos necesarios en el estudio (Freitas et al., 2004).

Una alternativa para identificar variables relevantes es el análisis de componentes principales (ACP), método estadístico de síntesis que identifica la información más importante. El procedimiento consiste en transformar el conjunto de variables originales en un nuevo conjunto de variables canónicas no relacionadas entre sí y ordenadas de forma decreciente respecto a la varianza. Así, los primeros componentes o variables canónicas contienen la mayor variabilidad de los datos originales y se descarta el resto de componentes y a las variables correlacionadas con éstos (Khattree y Naik, 2000; Barbosa et al., 2006).

El objetivo del presente estudio fue aplicar el ACP para determinar las variables fisicoquímicas y atributos sensoriales críticos durante el almacenamiento de frutos de mango cv. 'Haden' fresco cortado y almacenado a $5{ }^{\circ} \mathrm{C}$.

\section{MATERIALES Y MÉTODOS}

\section{Preparación de muestras}

Frutos de Mango del cv. 'Haden' se obtuvieron de huertas experimentales de Huatabampo, Sonora, México, y fueron procesados al alcanzar un contenido de sólidos solubles totales (SST) de 16 a $17 \%$. Los mangos enteros fueron lavados con agua clorada $\left(150 \mu \mathrm{g} \mathrm{L}^{-1}\right)$, pelados y cortados en cubos de $2.5 \mathrm{~cm}$ por arista aproximadamente. Los cubos se sanitizaron por inmersión en agua clorada (50 $\mathrm{gg} \mathrm{L}^{-1}$ durante $2 \mathrm{~min}$ ) y el agua residual superficial se eliminó con una centrífuga manual marca Essoreuse ${ }^{\circledR}$ modelo 1642 Jakarta, Indonesia. El producto cortado (80 g) fue envasado en bandejas de polietileno de $250 \mathrm{~cm}^{3} \mathrm{con}$ tapa (232 bandejas en total) y almacenado durante $14 \mathrm{~d}$ a $5{ }^{\circ} \mathrm{C}$. Cada $2 \mathrm{~d}$ se analizaron 29 bandejas, 20 de las cuales se utilizaron para la evaluación sensorial y 9 para evaluar los cambios en color, firmeza, SST y pH. Una bandeja fue la unidad experimental y se usaron tres repeticiones por tratamiento.

\section{Evaluación de cambios fisicoquímicos}

Al inicio del experimento y luego en cada muestreo durante el almacenamiento, se midió el cambio en color en cuatro cubos de cada una de cuatro bandejas destinadas para tal fin, durante todo el experimento. La evaluación se hizo con un colorímetro Minolta Croma Meter CR300® (Minolta Corp., Ramsey, NJ, USA) de acuerdo con el sistema CIE (L*, a* y b*) con D65 como iluminante de referencia y con un ángulo del observador de $10^{\circ}$. Se determinaron los valores de $\mathrm{L}^{*}$ (luminosidad), $\mathrm{a}^{*}$ (componente roja del color) y $\mathrm{b}^{*}$ (componente amarilla del color), y con ellos se calcularon las variables croma $\left(\mathrm{C}^{*}\right)$, ángulo de matiz $\left(\mathrm{h}^{\circ}\right)$ y diferencia total de color $\left(\Delta \mathrm{E}^{*}\right)$.

Las mediciones de firmeza, SST y $\mathrm{pH}$ se realizaron por triplicado en cada bandeja. La firmeza se determinó en los cubos de mango con un penetrómetro Chatillón modelo TCM200® (Ametek Instruments, Largo, FL, USA) provisto con un punzón de $1.2 \mathrm{~mm}$ de diámetro, y los resultados se expresaron como la fuerza $(\mathrm{N})$ requerida para penetrar el tejido a una velocidad de $8 \mathrm{~mm} \mathrm{~s}^{-1}$. El porcentaje de sólidos solubles (SST) se determinó por refractometría $\left({ }^{\circ} \mathrm{Bx}\right)$ con un refractómetro digital marca ATAGO modelo PR-101® (Atago Co. Ltd., Tokyo, Japan). El pH se midió se midió con la técnica de inmersión del electrodo, con un potenciómetro digital marca Corning modelo Pinnacle 545® (Corning Medical and Instrument, U.K.), calibrado con amortiguadores de pH 7 y 4; esta medición se hizo en 20 g de muestra homogeneizada.

\section{Evaluación de cambios sensoriales}

Para integrar el panel sensorial, los jueces fueron preseleccionados con base en su habilidad en la detección e identificación de olores y en su capacidad discriminante de los atributos de color, dulzor, acidez y textura 
evaluados sobre muestras estándar, preparadas de acuerdo con los procedimientos establecidos por la Agencia Española de Normalización (AENOR, 1997). La selección final de jueces se hizo con base en los resultados de un análisis secuencial (Meilgaard et al., 1999); una vez seleccionados, los jueces generaron una lista individual de términos descriptores del deterioro del mango fresco cortado, en muestras tomadas a diferentes tiempos de almacenamiento previamente preparadas. La selección final de descriptores se hizo con el método previamente reportado por Moskowitz (1983). Los términos así generados se utilizaron para integrar la planilla de evaluación sensorial.

El entrenamiento del panel se realizó mediante la evaluación de muestras con diferente grado de deterioro. Los resultados de cada sesión de entrenamiento se analizaron mediante un análisis de varianza con un diseño completamente al azar con arreglo factorial, para determinar diferencias significativas debidas a los factores tiempo de almacenamiento y panelista. El entrenamiento se concluyó luego de 12 sesiones, cuando no se observaron diferencias significativas entre panelistas. El panel quedó conformado por 10 jueces (seis mujeres y cuatro hombres).

La evaluación sensorial se llevó a cabo en cabinas individuales provistas de luz incandescente en un área con temperatura controlada $\left(25 \pm 2{ }^{\circ} \mathrm{C}\right)$. Cada juez recibió las muestras en su envase original en orden aleatorio, y debidamente codificadas con números aleatorios de tres dígitos. El primer atributo a evaluar fue el olor característico a mango, seguido de los atributos de apariencia, brillo, color, oscurecimiento, sabor, textura, acidez y dulzor. La intensidad de cada atributo se indicó con una marca colocada sobre una escala lineal no estructurada de $10 \mathrm{~cm}$, con los términos ancla ("excesivo" y "ninguno" para oscurecimiento y acidez, y "muy malo" y "óptimo" para el resto de los atributos) ubicados a cada extremo de la línea. La cuantificación de las respuestas se hizo midiendo la distancia (en $\mathrm{cm}$ ) desde el ancla izquierda, hasta la marca señalada por el panelista. Todas las muestras se mantuvieron a una temperatura de $12{ }^{\circ} \mathrm{C}$ durante la evaluación.

\section{Análisis estadístico}

Los datos obtenidos de la evaluación sensorial se analizaron mediante estadística descriptiva y análisis de varianza con un diseño completamente aleatorizado, para determinar diferencias significativas en los atributos sensoriales respecto al tiempo de almacenamiento, seguido de una prueba de comparación de medias por el método de Tukey $(\mathrm{P} \leq 0.05)$ (datos no mostrados).
Adicionalmente se hizo un análisis de regresión para determinar la tasa de cambio de los atributos sensoriales en función de las variables fisicoquímicas.

Además, se llevó a cabo un análisis de componentes principales (ACP) con la metodología propuesta por Rencher (2002), previa validación de supuestos de normalidad multivariada. El ACP parte de una matriz $Y$, de dimensiones $n \times p$, donde $p$ corresponde al número de variables observadas en $n$ individuos o muestras. La matriz $Y$ debe ser estandarizada de manera que permita obtener medias nulas y varianzas unitarias por variable. La matriz $X$ es el resultado de la transformación siguiente:

$$
X_{i j}=\frac{y_{i j}-\bar{y}_{j}}{s_{j} \sqrt{n}}
$$

Donde $\bar{y}_{j}$ y $s_{j}$ son la media y la desviación estándar de cada variable.

Posteriormente se determinaron los valores propios y vectores de la matriz $X^{\prime} X$. Los valores propios corresponden a la varianza de los valores en cada componente y los elementos de los vectores propios corresponden a las coordenadas en el espacio inicial que dan la dirección de los componentes principales. La ecuación matricial del ACP utilizada fue:

$$
Z=X U
$$

Donde $Z$ es la matriz de componentes principales; $X$, la matriz de valores iniciales estandarizados, y $U$ la matriz de vectores propios de la matriz $X^{\prime} X$ o matriz de correlación $R$.

En el análisis de correlación entre las variables se consideraron todos los resultados significativos a un valor $\mathrm{P} \leq 0.05$ y $\mathrm{P} \leq 0.01$. El grado de asociación entre las variables fisicoquímicas y los atributos sensoriales con los componentes principales, se consideró significativo a partir de un coeficiente de correlación de $0.70\left(r^{2} \geq 0.70\right)$.

Los análisis se llevaron a cabo con el paquete $\begin{array}{lllll}\text { estadístico } & \text { STATGRAPHICS Plus }{ }^{\circledR} & \text { Ver. } & 5.1\end{array}$ (Manugistics, Inc. Rockville, MD, USA) (Statgraphics, 2000). 


\section{RESULTADOS Y DISCUSIÓN}

\section{Análisis de componentes principales (ACP)}

Para llevar a cabo el ACP se requiere que los datos correspondan a una variable aleatoria con distribución normal multivariada; en la prueba analítica correspondiente se obtuvo un valor de probabilidad de error de 0.049 para los datos sensoriales y de 0.037 para los datos fisicoquímicos, lo que permite validar este supuesto.

\section{Variables fisicoquímicas}

El ACP resulta importante si existe correlación entre las variables, lo que indica que existe información redundante y pocos factores explican gran parte de la variabilidad total. En el Cuadro 1 se muestra la correlación significativa $(\mathrm{P} \leq 0.05)$ entre $\Delta \mathrm{E}^{*}, \mathrm{C}^{*} \mathrm{y} \mathrm{h}^{\circ}$. En el ACP se extrajeron tres componentes principales con autovalores mayores que 1.0, que explicaron $66.9 \%$ de la variabilidad total entre las unidades experimentales; el resto de la variabilidad quedó repartida en cuatro componentes de menor importancia (Cuadro 2). De forma similar, en un estudio por Lin et al. (2006) el ACP permitió detectar dos componentes principales que explicaron $75 \%$ de la variabilidad total en muestras de pera (Pyrus communis L.) fresca cortada sometida a diferentes tratamientos de conservación.

Las variables fisicoquímicas que mostraron correlación alta con los componentes principales y con autovalores mayores que 1.0 , fueron $\mathrm{L}^{*}, \Delta \mathrm{E}^{*}$ y $\mathrm{C}^{*}$, que estuvieron asociadas al primer componente, así como $\mathrm{pH}$ y firmeza, asociadas a los componentes dos y tres, respectivamente (Cuadro 3).

\begin{tabular}{|c|c|c|c|c|c|c|c|}
\hline Variables fisicoquímicas & Valor L* & $\Delta \mathrm{E}^{*}$ & $\mathrm{C}^{*}$ & $\mathrm{~h}^{\circ}$ & Firmeza & SST & $\mathrm{pH}$ \\
\hline $\mathrm{L}^{*}$ & 1.00 & $-0.76^{*}$ & $0.54 * *$ & 0.19 & 0.03 & -0.08 & 0.05 \\
\hline$\Delta \mathrm{E}^{*}$ & & 1.00 & $-0.67 * *$ & $-0.37 *$ & 0.07 & -0.03 & -0.04 \\
\hline $\mathrm{C}^{*}$ & & & 1.00 & 0.25 & 0.17 & 0.07 & 0.02 \\
\hline $\mathrm{h}^{\circ}$ & & & & 1.00 & 0.04 & -0.01 & -0.08 \\
\hline Firmeza & & & & & 1.00 & -0.02 & -0.05 \\
\hline SST & & & & & & 1.00 & -0.13 \\
\hline $\mathrm{pH}$ & & & & & & & 1.00 \\
\hline$*, * * \mathrm{P} \leq 0.01, \mathrm{P} \leq 0.05$ & & & & & & & \\
\hline
\end{tabular}

Cuadro 2. Componentes principales, autovalores y porcentaje de la varianza total y acumulada correspondiente a los cambios fisicoquímicos en mango 'Haden' fresco cortado, obtenidos de acuerdo con Rencher (2002).

\begin{tabular}{lccc}
\hline Componente & Autovalor & Porcentaje de varianza total & Porcentaje de varianza acumulada \\
\hline 1 & $\mathbf{2 . 4 7}$ & 35.3 & 35.3 \\
2 & $\mathbf{1 . 1 6}$ & 16.7 & 52.0 \\
3 & $\mathbf{1 . 0 4}$ & 14.9 & $\mathbf{6 6 . 9}$ \\
4 & 0.92 & 13.2 & 80.1 \\
5 & 0.79 & 11.4 & 91.5 \\
6 & 0.42 & 6.0 & 97.5 \\
7 & 0.17 & 2.5 & 100.0 \\
\hline
\end{tabular}

Los números en negritas corresponden a los autovalores mayores que 1.0 (criterio utilizado para la extracción de los componentes principales), y al porcentaje de varianza acumulada explicado por los tres componentes principales elegidos. 
Cuadro 3. Coeficientes de correlación entre las variables fisicoquímicas y los componentes principales, correspondientes con los cambios fisicoquímicos en mango 'Haden' fresco cortado, obtenidos de acuerdo con Rencher (2002).

\begin{tabular}{|c|c|c|c|c|c|}
\hline \multirow{3}{*}{$\begin{array}{l}\text { Variables } \\
\text { fisicoquímicas }\end{array}$} & \multicolumn{5}{|c|}{ Componentes principales } \\
\hline & 1 & 2 & 3 & 4 & 5 \\
\hline & \multicolumn{5}{|c|}{ Coeficientes de correlación de Pearson } \\
\hline $\mathrm{L}^{*}$ & 0.84 & -0.17 & $-2.2 \mathrm{E}-02$ & $7.4 \mathrm{E}-02$ & -0.28 \\
\hline$\Delta \mathrm{E}^{*}$ & -0.92 & 4.6E-02 & 0.17 & $-1.1 \mathrm{E}-02$ & 5.9E-02 \\
\hline $\mathrm{C}^{*}$ & 0.82 & $9.5 \mathrm{E}-02$ & 0.11 & 0.21 & $-3.2 \mathrm{E}-02$ \\
\hline $\mathrm{h}^{\circ}$ & 0.48 & 0.23 & $3.0 \mathrm{E}-02$ & -0.59 & 0.60 \\
\hline Firmeza & 7.9E-02 & 0.26 & 0.92 & 0.23 & $8.5 \mathrm{E}-02$ \\
\hline SST & $3.9 \mathrm{E}-03$ & 0.66 & -0.39 & 0.56 & 0.28 \\
\hline $\mathrm{pH}$ & $3.6 \mathrm{E}-02$ & -0.76 & 4.3E-03 & 0.39 & 0.52 \\
\hline
\end{tabular}

Los números en negritas corresponden a las variables con mayor correlación ( $\mathrm{r}>0.70)$ con cada componente principal; $\mathrm{L}^{*}=$ componente $\mathrm{L}^{*}$ del color correspondiente a luminosidad (0 a 100$)$;

$\Delta \mathrm{E}^{*}=$ diferencia total de color $\left(\Delta E^{*}=\left[\left(L_{0}^{*}-L^{*}\right)^{2}+\left(a_{0}^{*}-a^{*}\right)^{2}+\left(b_{0}^{*}-b^{*}\right)^{2}\right]^{1 / 2}\right)$;

$\mathrm{C}^{*}=\operatorname{croma}\left(C^{*}=\left(a^{* 2}+b^{* 2}\right)^{l / 2}\right) ; \mathrm{h}^{\circ}=$ ángulo de matiz $\left(h^{\circ}=\operatorname{arctg}\left(b^{*} / a^{*}\right)\right)$;

SST $=$ porcentaje de sólidos solubles totales.

Estos resultados corresponden con los cambios observados en los componentes del color y las variables derivadas. El valor $\mathrm{L}^{*}$ mostró una disminución durante el almacenamiento, lo que concuerda con resultados obtenidos en cubos de mango de los cultivares 'Keitt', 'Kent' y 'Ataulfo' conservados a $5{ }^{\circ} \mathrm{C}$ (González-Aguilar et al., 2008). Resultados similares fueron obtenidos por Rattanapanone et al., (2001) en mango fresco cortado de los cultivares 'Kent' y 'Tommy Atkins' almacenados a temperatura ambiente. De acuerdo con González-Aguilar et al. (2004), el valor $\mathrm{L}^{*}$ es considerado un indicador de la frescura del producto, donde un valor alto de $\mathrm{L}^{*}$ en frutos frescos cortados se relaciona con una buena apariencia, mientras que valores bajos pueden indicar oscurecimiento en frutos cortados como el mango (González-Aguilar et al., 2000; González-Aguilar et al., 2004). Los resultados coinciden con los de Chauhan et al. (2006) quienes observaron una disminución de los valores $\mathrm{L}^{*} \mathrm{y} \mathrm{b}^{*}$ en mango fresco cortado.

Los caracteres derivados $\mathrm{C}^{*} \mathrm{y} \mathrm{h}^{\circ}$ indican la intensidad o saturación del color y el color verdadero, respectivamente. Así, la disminución de $\mathrm{C}^{*}$ indicó una menor saturación del color amarillo característico del mango fresco cortado, y la disminución en $\mathrm{h}^{\circ}$ una variación del color hacia un amarillo más claro. Este comportamiento corresponde con un cambio en el color, como consecuencia de la degradación de pigmentos y la pérdida de éstos durante la sanitización del producto cortado, lo cual resulta de la ruptura de células durante el corte (Vitti, 2003). Los resultados concuerdan con los de Talcott et al. (2005) quienes reportaron una disminución en $\mathrm{C}^{*}$ de mango fresco cortado almacenado a $5{ }^{\circ} \mathrm{C}$.
En cuanto a la firmeza, los resultados mostraron la importancia de esta variable entre los cambios fisicoquímicos evaluados. Según Rico et al. (2007), la pérdida de firmeza es indeseable en productos vegetales mínimamente procesados debido a que los consumidores asocian directamente la textura con la frescura del tejido vegetal, por lo que una apariencia blanda puede incrementar el rechazo del producto. En mango mínimamente procesado, un efecto significativo $(\mathrm{P} \leq 0.05)$ del almacenamiento en atmósfera controlada (AC) sobre la textura, fue observado por Chauhan et al. (2006). También González-Aguilar et al. (2008) indicaron una disminución significativa en la firmeza de cubos de mango de los cultivares 'Ataulfo', 'Kent' y 'Keitt', durante el almacenamiento a $5{ }^{\circ} \mathrm{C}$. De acuerdo con Martínez-Ferrer et al. (2002), la pérdida de firmeza observada en frutos precortados de piña (Ananas comosus L.) y mango, se debe a varios factores como pérdida de turgencia celular, pérdida del aire extracelular y vascular, y degradación de los constituyentes de la pared celular.

Para identificar las variables menos relevantes se analizó su correlación con los tres primeros componentes y con los de menor importancia (variables canónicas con autovalores menores que 1.0); las variables $\mathrm{h}^{\circ}$ y SST mostraron baja correlación $(r \leq 0.70)$ con los componentes principales (Cuadro 3 ).

En relación con el ángulo de matiz $\left(\mathrm{h}^{\circ}\right)$, GonzálezAguilar et al. (2008) indicaron que en mango el cambio en $\mathrm{h}^{\circ}$ está determinado por una baja temperatura de almacenamiento y el cultivar. La baja temperatura reduce la actividad de las polifenoloxidasas involucradas en este tipo de deterioro indeseable, y las diferencias entre las variedades de mango en contenidos de azúcares, ácidos 
orgánicos, vitamina $\mathrm{C}$, carotenoides y polifenoles, determinan diferencias en susceptibilidad al oscurecimiento (González-Aguilar et al., 2008). En el caso de SST, Beaulieu y Baldwin (2002) indicaron que su evolución en FVFC depende del producto y la temperatura durante y después del proceso. En el presente estudio, el poco cambio en esta variable podría estar determinado por la baja temperatura de almacenamiento.

\section{Atributos sensoriales}

Se observó una correlación significativa $(\mathrm{P} \leq 0.01$ y $\mathrm{P} \leq$ 0.05) entre algunos atributos sensoriales, por lo que la aplicación del ACP permitió resumir la variabilidad total observada entre unidades experimentales (Cuadro 4). En este caso se identificaron tres componentes principales (con autovalores mayores que 1.0) que explicaron $68.3 \%$ de la variabilidad (Cuadro 5). El análisis de las variables correlacionadas con cada variable canónica (Cuadro 6) indicó que los atributos con mayor cambio durante el almacenamiento del mango 'Haden', fueron apariencia, sabor y color, que tuvieron una alta correlación $(\mathrm{r}>$ 0.70) con el primer componente; el oscurecimiento mostró alta correlación ( $\mathrm{r}>0.70)$ con el segundo componente, y el brillo con el tercer componente principal. El color (asociado con el componente principal 3) fue considerado relevante por mostrar un coeficiente de correlación muy cercano al valor de referencia $(\mathrm{r}=$ $0.70)$.

Estos resultados corresponden con los obtenidos por Lin et al. (2006) en pera fresca cortada, donde los cambios relacionados con sabor, dulzor, olor característico y textura se asociaron con el primer componente principal, mientras que la apariencia y el color mostraron mayor correlación con el componente dos; es decir, tales atributos mostraron los mayores cambios en el producto.

En relación con el sabor, los resultados concuerdan con los de Monteiro et al. (2004) quienes reportaron una disminución lineal de las notas del sabor característico de mango fresco cortado, al transcurrir el tiempo de almacenamiento. Asimismo, De Souza et al. (2006) encontraron que mangos precortados naturalmente madurados presentaron mejor sabor y preferencia del consumidor que los mangos madurados con etileno, comportamiento que indica el efecto del manejo sobre los atributos de la calidad sensorial.

\begin{tabular}{|c|c|c|c|c|c|c|c|c|c|}
\hline Atributos sensoriales & Apariencia & Brillo & Color & Osc. & Olor & Textura & Sabor & Acidez & Dulzor \\
\hline Apariencia & 1.00 & $0.57 * *$ & $0.61 * *$ & -0.28 & $0.42 * *$ & 0.27 & $0.48 * *$ & $-0.36^{*}$ & $0.44 * *$ \\
\hline Brillo & & 1.00 & $0.44 * *$ & 0.05 & $0.46^{* *}$ & 0.06 & 0.26 & -0.13 & 0.18 \\
\hline Color & & & 1.00 & -0.21 & 0.26 & $0.42 * *$ & $0.32 * *$ & -0.19 & $0.35^{*}$ \\
\hline Osc. & & & & 1.00 & 0.24 & 0.01 & -0.19 & -0.16 & -0.07 \\
\hline Olor & & & & & 1.00 & $0.31 *$ & 0.29 & $-0.44 * *$ & 0.29 \\
\hline Textura & & & & & & 1.00 & $0.46^{* *}$ & $-0.31 *$ & $0.30^{*}$ \\
\hline Sabor & & & & & & & 1.00 & $-0.33^{*}$ & $0.54 * *$ \\
\hline Acidez & & & & & & & & 1.00 & $-0.49 * *$ \\
\hline Dulzor & & & & & & & & & 1.00 \\
\hline
\end{tabular}

$*, * * \mathrm{P} \leq 0.05, \mathrm{P} \leq 0.01$. Osc. $=$ oscurecimiento superficial.

Cuadro 5. Componentes principales, autovalores y porcentaje de la varianza total y acumulada correspondiente a los atributos sensoriales evaluados en mango 'Haden' fresco cortado, obtenidos de acuerdo con Rencher (2002).

\begin{tabular}{lccc}
\hline Componente & Autovalor & Porcentaje de varianza total & Porcentaje de varianza acumulada \\
\hline 1 & $\mathbf{3 . 5 4}$ & 39.36 & 39.36 \\
2 & $\mathbf{1 . 4 1}$ & 15.62 & 54.98 \\
3 & $\mathbf{1 . 1 9}$ & 13.30 & $\mathbf{6 8 . 2 9}$ \\
4 & 0.819 & 9.10 & 77.39 \\
5 & 0.599 & 6.66 & 84.05 \\
6 & 0.540 & 6.00 & 90.05 \\
7 & 0.382 & 4.25 & 94.30 \\
8 & 0.267 & 2.96 & 97.26 \\
9 & 0.246 & 2.74 & 100.00 \\
\hline
\end{tabular}

Los números en negritas corresponden a los autovalores mayores que 1.0 (criterio utilizado para la extracción de los componentes principales) y al porcentaje de varianza acumulada explicada por los tres componentes elegidos. 
Cuadro 6. Matriz de componentes y atributos sensoriales asociados, obtenida de acuerdo con Rencher (2002).

\begin{tabular}{lccccc}
\hline \multirow{2}{*}{ Atributos sensoriales } & \multicolumn{5}{c}{ Componentes principales } \\
\cline { 2 - 5 } & 1 & 2 & 3 & 4 & 5 \\
\hline Apariencia & $\mathbf{0 . 8 1}$ & -0.27 & 0.26 & -0.14 & $-9.7 \mathrm{E}-02$ \\
Brillo & 0.58 & $2.4 \mathrm{E}-03$ & $\mathbf{0 . 7 0}$ & $-4.7 \mathrm{E}-02$ & 0.19 \\
Color & $\mathbf{0 . 6 9}$ & -0.32 & 0.19 & 0.34 & -0.36 \\
Oscurecimiento & -0.11 & $\mathbf{0 . 8 7}$ & 0.14 & 0.16 & $5.9 \mathrm{E}-02$ \\
Olor & 0.63 & 0.48 & 0.27 & $3.3 \mathrm{E}-02$ & $9.4 \mathrm{E}-02$ \\
Textura & 0.57 & $8.1 \mathrm{E}-02$ & -0.41 & 0.66 & $3.0 \mathrm{E}-02$ \\
Sabor & $\mathbf{0 . 7 1}$ & -0.15 & -0.34 & $-5.5 \mathrm{E}-02$ & 0.52 \\
Acidez & -0.59 & -0.45 & 0.29 & 0.29 & 0.37 \\
Dulzor & 0.68 & $2.7 \mathrm{E}-02$ & -0.37 & -0.37 & $-1.0 \mathrm{E}-02$ \\
\hline Los números en negritas corresponden a las variables con mayor correlación (r $\geq 0.70)$ con cada componente principal.
\end{tabular}

Según Aked (2000), la apariencia es el atributo más importante en FVFC. En ésta se involucran aspectos como tamaño, forma, marchitamiento, pérdida de brillantez y coloraciones indeseables. Al respecto Chauhan et al. (2006) consideran que la estabilidad del color en mango mínimamente procesado está determinada por la susceptibilidad al oscurecimiento y pérdida de pigmentos. Poubol e Izumi (2005) reportaron que la vida en anaquel de mangos 'Carabao' precortados, con base en oscurecimiento y apariencia acuosa, fue de $6 \mathrm{~d}$ a $5{ }^{\circ} \mathrm{C}$ y de $4 \mathrm{~d} \mathrm{a} 13{ }^{\circ} \mathrm{C}$.

El resto de los atributos sensoriales mostraron baja correlación $\left(\mathrm{r}<0^{0}\right.$.70) con los tres componentes principales, o bien presentaron mayor asociación con los componentes de menor relevancia (con autovalores menores a 1.0), lo que indica que estos atributos fueron poco útiles para explicar las diferencias entre unidades experimentales debidas al tiempo de almacenamiento.

Los resultados aquí obtenidos en relación con la apariencia, brillo, color y oscurecimiento, fueron consistentes con los cambios observados en los valores de $\mathrm{L}^{*}, \mathrm{C}^{*} \mathrm{y} \Delta \mathrm{E}^{*}$ del color.

Respecto a la textura evaluada sensorialmente, Salvador et al. (2007) indicaron que el almacenamiento a bajas temperaturas disminuye significativamente la pérdida de textura, debido a la reducción de la actividad de las enzimas pectinmetilesterasas (PME) y poligalacturonasas (PG) que participan en la eliminación de las pectinas de la lámina media. Estudios previos hechos por GonzálezAguilar et al. (2008), indican que la firmeza disminuyó significativamente en tres cultivares de mango fresco cortado almacenados a $5{ }^{\circ} \mathrm{C}$. Cienfuegos et al. (2004) y Plotto et al. (2006) también observaron disminución significativa de la firmeza con el trascurso del tiempo en mango de la variedad 'Kent', que corresponde con el cambio en la textura evaluada sensorialmente.

\section{Análisis de regresión}

Mediante análisis de regresión es posible obtener la tasa de deterioro de una característica, como la pendiente de la recta que describe su trayectoria. El comportamiento respecto al tiempo (x) de almacenamiento de los atributos sensoriales y cambios fisicoquímicos (y), se determinó mediante regresión lineal simple y el coeficiente de determinación $\left(\mathrm{R}^{2}\right)$ se consideró como criterio de ajuste (Cuadro 7). La calificación inicial promedio del atributo, evaluado por el panel sensorial entrenado mediante una escala no estructurada de $10 \mathrm{~cm}(0=$ muy malo, $10=$ óptimo), correspondió al intercepto de la ecuación de regresión. El mayor valor inicial correspondió al olor característico, y el menor al brillo.

Los coeficientes de determinación $\left(\mathrm{R}^{2}\right)$ mostraron valores de 0.67 a 0.90 en los atributos sensoriales, y de 0.79 a 0.96 en las variables fisicoquímicas. Estos resultados indican un ajuste adecuado del modelo de regresión lineal a la cinética de los cambios evaluados en mango 'Haden' fresco cortado almacenado a $5{ }^{\circ} \mathrm{C}$, lo que corresponde con los resultados de Chauhan et al. (2006) y Salinas-Hernández et al. (2009), quienes observaron una cinética de orden cero en los cambios fisicoquímicos de mango fresco cortado de los cvs. 'Arka anmol' y 'Kent'.

La tasa de cambio obtenida para cada atributo sensorial indicó que el brillo característico del fruto recién procesado, presentó cambios inmediatos después del proceso. Este comportamiento coincidió con el observado en la evolución de los atributos durante el almacenamiento, donde la mayor velocidad de cambio (pendiente de la recta) se obtuvo en el brillo (0.2456), seguido de la firmeza (0.1561) y luego el dulzor (0.1512); en tanto que los atributos con menor velocidad de cambio fueron el sabor característico (0.098) y el olor (0.0626). 
Cuadro 7. Ecuaciones de regresión y coeficientes de determinación $\left(\mathbf{R}^{2}\right)$ de atributos sensoriales y variables físicoquímicas evaluadas en mango 'Haden' fresco cortado almacenado a $5{ }^{\circ} \mathrm{C}$.

\begin{tabular}{llc}
\hline Atributos y variables & \multicolumn{1}{c}{ Ecuación de regresión } & Coeficiente de determinación $\left(\mathrm{R}^{2}\right)$ \\
\hline Atributos sensoriales & Dulzor $=9.2609-0.1512 \mathrm{x}$ & 0.67 \\
& Textura $=9.0291-0.1561 \mathrm{x}$ & 0.75 \\
& Brillo $=8.9739-0.2456 \mathrm{x}$ & 0.89 \\
& Color $=9.4491-0.106 \mathrm{x}$ & 0.82 \\
& Sabor $=9.1222-0.098 \mathrm{x}$ & 0.87 \\
& Olor $=9.5084-0.0626 \mathrm{x}$ & 0.90 \\
& Apariencia $=9.3407-0.1049 \mathrm{x}$ & 0.79 \\
Variables fisicoquímicas & $\mathrm{L}^{*}=59.807-0.4692 \mathrm{x}$ & 0.95 \\
& $\mathrm{a}^{*}=5.0462-0.4356 \mathrm{x}$ & 0.87 \\
& $\mathrm{~b}^{*}=37.048-0.6454 \mathrm{x}$ & 0.87 \\
& $\mathrm{C}^{*}=46.279-0.6558 \mathrm{x}$ & 0.87 \\
& $\mathrm{~h}^{\circ}=89.691-0.4374 \mathrm{x}$ & 0.79 \\
& $\Delta \mathrm{E}^{*}=8.3496+0.2352 \mathrm{x}$ & 0.87 \\
& $\mathrm{SST}=13.465+0.0414 \mathrm{x}$ & 0.96 \\
& Firmeza $=0.3243-0.010 \mathrm{x}$ & 0.92 \\
$\mathrm{pH}=3.9156+0.0171 \mathrm{x}$ & 0.86 \\
\hline
\end{tabular}

El intercepto en los cambios sensoriales corresponde a la calificación inicial promedio del atributo, evaluado por el panel sensorial entrenado y con uso de una escala no estructurada de $10 \mathrm{~cm}(0=$ muy malo, $10=$ óptimo $)$, en las variables fisicoquímicas corresponde al valor inicial de la variable evaluada instrumentalmente. La pendiente corresponde a la tasa de cambio del atributo sensorial o la variable fisicoquímica evaluada. El valor de $x$ corresponde al tiempo (días); Los componentes del color corresponden al cambio de verde a rojo $\left(-a^{*}\right.$ a $\left.+a^{*}\right)$, de azul a amarillo $\left(-b^{*}\right.$ a $\left.+b\right)$ y de luminosidad $\left(\mathrm{L}^{*}=0\right.$ a $\left.\mathrm{L}^{*}=100\right)$; Los valores derivados $\mathrm{C}^{*}, \mathrm{~h}^{\circ}$ y $\Delta \mathrm{E}^{*}$ corresponden a croma $\left(C^{*}=\left(a^{* 2}+b^{* 2}\right)^{1 / 2}\right.$; ángulo de matiz $\left(h^{\circ}=\operatorname{arctg}\left(b^{*} / a^{*}\right)\right.$ y diferencia total de color $\left(\Delta E^{*}=\left[\left(L_{0}^{*}-L^{*}\right)^{2}+\left(a^{*}{ }_{0}-a^{*}\right)^{2}+\left(b^{*}{ }_{0}-b^{*}\right)^{2}\right]^{1 / 2}\right)$.

Estos resultados indican diferencias importantes en la velocidad con que se pierden los atributos deseables en el mango fresco cortado, pues el brillo mostró una velocidad de pérdida cuatro veces mayor que el atributo con mayor permanencia en el producto que resultó ser el olor característico. Estos resultados son importantes, ya que de acuerdo con Ragaert et al. (2004), el olor, el sabor y la textura determinan la decisión de compra de los productos vegetales frescos cortados. En cuanto a las variables fisicoquímicas, la mayor tasa de cambio se obtuvo para croma $\left(C^{*}\right)(0.656)$ y la menor para firmeza $(0.010)$.

Los cambios aquí observados confirmaron que la apariencia general y el color constituyen los cambios sensoriales y fisicoquímicos más importantes en FVFC en general y del mango fresco cortado en particular, debido a que se asocian con la frescura del producto (colores vivos y uniformes), y con un aspecto turgente acompañado de un brillo característico, como señalaron Péneau et al.( 2007).

\section{CONCLUSIONES}

El análisis de componentes principales permitió identificar las variables fisicoquímicos y atributos sensoriales críticos durante el almacenamiento de mango fresco cortado almacenado a $5{ }^{\circ} \mathrm{C}$. Estos cambios pueden ser utilizados como indicadores del deterioro en estudios de vida de anaquel del producto. La tasa de deterioro de los atributos sensoriales del mango fresco cortado indicó que el brillo, atributo característico del fruto recién procesado, tuvo una tasa de cambio cuatro veces mayor que el olor y éste último el atributo es el de mayor duración en el producto. Las variables fisicoquímicas con mayores cambios fueron las relacionadas con el color.

\section{BIBLIOGRAFÍA}

AENOR, Asociación Española de Normalización y Certificación (1997) Análisis Sensorial. Asociación Española de Normalización y Certificación. Tomo 1: Alimentación. Recopilación de normas UNE. AENOR, Madrid, España. 253 p.

Aked J (2000) Fruits and vegetables. In: The Stability and Shelf-life of Food. D Kilcast, P Subramaniam (eds). Woodhead Publishing Ltd. and CRC Press LLC. Boca Raton, Fla., USA. pp:249-278.

Alzamora S M, A López-Malo, M S Tapia (2000) Overview on minimallly processed fruits and vegetables. In: Minimally Processed Fruits and Vegetables. Fundamental Aspects and Applications. SM Alzamora, A López-Malo, MS Tapia (eds). Aspen Publ. Gaitherburg, MD, USA. pp:2-9.

Barbosa L, P S Lopes, A J Regazzi, S E Facioni, G Robledo de Almeida Torres (2006) Avaliação de características de qualidade da carne de suínos por meio de componentes principais. Rev. Bras. Zootec. 35:1639-1645.

Beaulieu J C, E A Baldwin (2002) Flavor and aroma of fresh-cut fruits and vegetables. In: Fresh-cut Fruits and Vegetables. Science, Technology and Market. O Lamikanra. (ed). CRC Press. Boca Raton, Fla, USA: pp:391-425. 
Cienfuegos Z E, H S García, M Mata, G B Tovar (2004) Aceleración de la maduración de mango 'Kent' refrigerado. Rev. Fitotec. Mex. 27:359-366.

Chauhan O P, P S Raju, D K Dasgupta, R Shylaja, R Sudhakar, A S Bawa (2006) Modified/controlled atmosphere storage of minimally processed mango slices (Var. Arka anmol). Amer. J. Food Technol. 1:105-116.

De Souza B S, T J O'Hare, J F Durigan, P S de Souza (2005) Impact of atmosphere, organic acids, and calcium on quality of freshcut 'Kensington' mango. Posthar. Biol. Technol. 42:161-167.

Freitas R T F, T M Gonçalves, A I G Oliveira (2004) Avaliação de carcaças de suínos da raça Large White utilizando medidas convencionais. Rev. Bras. Zootec. 33:2037-2043.

Gil M I, E Aguayo, A A Kader (2006) Quality changes and nutrient retention in fresh-cut versus whole fruits during storage. J. Agric. Food Chem. 54:4284-4296.

González-Aguilar G A, C Wang, J G Buta (2000) Maintaining quality of fresh-cut mangoes using antibrowning agents and modified atmosphere packaging. J. Agric. Food Chem. 48:4204-4208.

González-Aguilar G A, S Ruiz-Cruz, R Cruz-Valenzuela, A Rodríguez-Félix, C Y Wang (2004) Physiological and quality changes of fresh-cut pineapple treated with antibrowning agents. Food Sci. Technol.-Lebensmittel-Wissenschaft and Technologie 37:369-376.

González-Aguilar G, J Celis, R R Sotelo-Mundo, L A de la Rosa, J Rodrigo-García, E Álvarez-Parrilla (2008) Physiological and biochemical changes of different fresh-cut mango cultivars stored at $5{ }^{\circ}$ C. Intl. J. Food Sci. Technol. 43:91-101.

Khattree R, D N Naik (2000) Multivariate data reduction and discrimination with SAS software. SAS Institute Inc. Cary, NC., USA. 574 p.

Lin D S, S W Leonard, C Lederer, M G Traber, Y Zhao (2006) Retention of fortified vitamin E and sensory quality of fresh-cut pears by vacuum impregnation with honey. J. Food Sci. 71: 553-559.

Martínez-Ferrer M, Harper C, F Pérez-Muñoz, M Chaparro (2002) Modified atmosphere packaging of minimally processed mango and pineapple fruits. J. Food Sci. 67:3365-3371.

Meilgaard M, G V Civille, B T Carr (1999) Sensory Evaluation Techniques. CRC Press. Boca Raton, FLA, USA. pp:199-222.

Monteiro V B B, E E Nunes, F F Vilela, L C de Oliveira, E V de Barros, C A H Romaniello (2004) Avaliacao da qualidade de mangas 'Tommy Atkins' minimamente processadas. Rev. Bras. Frut. 26:540-543.

Moskowitz H R (1983) Product Testing and Sensory Evaluation of Foods. Food \& Nutrition Press, Trumbull, Conn., USA. pp:2430.
Péneau S, P B Brockhoff, E Hoehn, F Escher, J Nuessli (2007) Relating consumer evaluation of apple freshness to sensory and physico-chemical measurements. J. Sensory Studies 22:313 335.

Plotto A J, Bai J, J Narciso, K Brecht, E A Baldwin (2006) Ethanol vapor prior to processing fresh-cut mango storage by decreasing spoilage, but does not always delay ripening. Postharv. Biol. Technol. 39:134-145.

Poubol J, H Izumi (2005) Shelf life and microbial quality of fresh-cut mango cubes stored in high $\mathrm{CO} 2$ atmospheres. J. Food Sci. 70:M69-M74.

Qüesta A G, S C Rodríguez, S Generoso, R M Soliba (2007) El análisis sensorial. Herramienta para evaluar calidad en vegetales mínimamente procesados. In: Avances Tecnológicos en el Procesado Mínimo Hortofrutícola, Aspectos Nutricionales y Sensoriales. G A González-Aguilar, J F Ayala-Zavala (eds) Cartagena, Murcia. España. pp:63-70.

Ragaert P, W Verbeke, F Devlieghere, J Debevere (2004) Consumer perception and choice of minimally processed vegetables and packaged fruits. Food Qual. Prefer. 15:259-270.

Rencher A C (2002) Methods of Multivariate Analysis. 2nd ed. John Wiley \& Sons. New York, USA. 708 p.

Rico D, A B Martín-Diana, J M Baratb, C Barry-Ryana (2007) Extending and measuring the quality of fresh-cut fruit vegetables: a review. Trends Food Sci. Technol. 18:373-386.

Rivera-López J, F A Vázquez-Ortiz, J F Ayala-Zavala, R R SoteloMundo, G A González-Aguilar (2005) Cutting shape and storage temperature affect overall quality of fresh-cut papaya cv. 'Maradol'. J. Food Sci. 70:482-489.

Salinas-Hernández R M, M E Pirovani, F Ulín-Montejo, G A González-Aguilar (2009) ¿Se puede predecir la vida de anaquel de un alimento? El caso de frutos mínimamente procesados. Rev. Ind. Alim. 31:25-34.

Salvador A, P Varela, S M Fiszman (2007) Consumer acceptability and shelf life of "Flor de invierno" pears (Pyrus communis L.) under different storage conditions. J. Sensory Studies 22:243255.

Sancho J, E Bota, J J De Castro (2002) Introducción al Análisis Sensorial de los Alimentos. Ed. Alfaomega. Zaragoza, España. pp:119-129.

Statgraphics (2000) Statgraphics Plus Ver.5.1 Herndon, VIR Stat Point, Inc. Manugistics, Inc. Rockville, MD, USA.

Talcott S T, J P Moore, A J Lounds-Singleton, S S Percival (2005) Ripening associated phytochemical changes in mangos (Mangifera indica) following thermal quarantine and lowtemperature storage. J. Food Sci. 70:337-341.

Vitti M C D (2003) Comportamento da beterraba minimamente processada em diferentes espessuras de corte. Hort. Bras. 21:623-626. 